\title{
Characteristics of Spinach Chloroplast Envelope, Thylakoid and Stroma Polypeptides as Revealed by Triton X-114 Phase Partition
}

\author{
Paul-André Siegenthaler and Nicole Dumont \\ Laboratoire de Physiologie végétale, Université de Neuchâtel, 20, rue de Chantemerle, \\ CH-2000 Neuchâtel, Switzerland
}

\begin{abstract}
Comparison of the SDS-PAGE profiles of the spinach chloroplast stroma, thylakoid and envelope membranes shows that several polypeptides have the same electrophoretic mobility. To simplify these somewhat complex electrophoretic profiles and to verify whether the polypeptides having similar electrophoretic mobility are identical, we used Triton X-114 phase partition to obtain a separation of the polypeptides according to their relative hydrophobicity. The stroma polypeptides partitioned essentially in the aqueous phase. About half of the thylakoid and envelope membrane polypeptides were exclusively recovered in either one of the two phases. Therefore, the phase partitioning of membrane polypeptides proved to be useful, as the organic phase contained true intrinsic polypeptides, while the aqueous phase was composed of peripheral ones and stroma components. Particularly interesting was the release of the RubisCO large subunit known to copurify with the envelope membranes. Additional experimental approaches were used (immunology, proteosynthesis in organello) to further characterize proteins which had apparent ambiguous phase partitioning. Here, we show that Triton X-114 is an excellent tool to unmask polypeptides having identical electrophoretic mobility but different behaviour towards this detergent; its use leads to a clarification of the polypeptide SDS-PAGE profiles of chloroplast membranes.
\end{abstract}

Key words: Electrophoretic separation - Envelope membranes - Hydrophobicity - Immunology - Spinach chloroplast polypeptides - Triton X-114.

Chloroplasts from higher plants are made up of six different compartments (the outer and inner envelope membranes, the intermembrane space, the stroma, the thylakoid membranes and the thylakoid lumen), each of which having its own functions and a distinct set of polypeptides. The envelope serves as a boundary between the cytosol and the chloroplast and is the site of many enzymatic activities on top of its role as a selective barrier for the movement of molecules in and out of the chloroplast (Douce et al. 1984). The envelope, the polypeptides of which represent only $1-2 \%$ of the total chloroplast proteins, is composed of two membranes with different densities and permeabilities (Douce et al. 1984).

Abbreviations: Mops, (3-[N-morpholino]propanesulfonic acid); PMSF, phenylmethane sulfonyl fluoride; PAGE, polyacrylamide gel electrophoresis; IEF, isoelectric focusing; pI, isoelectric point; TX-114, Triton X-114 (alkylphenylpolyethyleneglycol); TX100 , Triton X-100; T, thylakoid; S, stroma; IM, inner membrane; $\mathrm{OM}$, outer membrane; RubisCO, ribulose 1,5-bisphosphate carboxylase/oxygenase; Mr, apparent molecular weight.
The electrophoretic analysis of the inner and outer envelope membranes in one-dimension shows that each membrane has its own set of polypeptides which differ from those of the thylakoid and the stroma (Joyard et al. 1982, Werner-Washburne et al. 1983). However, the two envelope membranes are linked together by contact sites as shown by freeze-fracture electron microscopy (Cline et al. 1985) and freeze-fracture and freeze-substitution combined with ultrathin sectioning (Cremers et al. 1988). Upon separation of the two membranes in hypertonic medium followed by their purification, these contact sites give rise to stroma-containing vesicles migrating with the inner membrane during centrifugation. The characterization of the two envelope membranes is thus complicated by the presence of stroma. Furthermore, even though the envelope membrane purification methods have been greatly improved, it is not possible to obtain pure fractions and the electrophoretic profiles may not be totally representative of the subchloroplast fractions studied.

Moreover, they are difficult to analyze as different polypeptides may display the same electrophoretic mobility and 
migrate as a unique Coomassie blue band.

In an attempt to go deeper into the characterization of the two envelope membrane polypeptides and to assess and identify the source of any cross-contamination that may occur during the purification of the two membranes, we have used TX-114 temperature-induced phase partition as described by Bordier (1981). TX-114 is the only detergent of the TX-100 series which forms clear micellar solutions in water at low temperature $\left(4^{\circ} \mathrm{C}\right)$ and separates above $20^{\circ} \mathrm{C}$ into two phases, respectively enriched and depleted in detergent. The TX-114 phase partition has been used successfully by Bricker and Sherman (1982) on maize thylakoid membranes and, more recently, by Kjellbom et al. (1989) on spinach leaf plasma membrane. Here, we have applied the TX-114 phase partition method to chloroplast envelope membranes with the specific aim of clarifying their complex electrophoretic profiles. For comparison, we have included stroma and thylakoid membrane electrophoretic separations in order to better detect possible contamination by these fractions. This technique gives rise to a preliminary separation of the proteins according to their hydrophobic/hydrophilic character while keeping them in a membrane-like environment, thus preserving their native form. This treatment, followed by electrophoresis, enables to separate several polypeptides of similar $\mathrm{Mr}$ but of different behaviour towards TX-114 phase partition and helps to identify polypeptides of different origin. However, due to ambiguous phase partitioning and to polypeptide comigration that may still exist, it was imperative to include other criteria based on results from 2-D electrophoresis, immunology and proteosynthesis in organello, to differentiate between those polypeptides that could have been otherwise mistaken for others. The present study extends the results already presented in 1988 (Dumont et al.) and 1989 (Dumont and Siegenthaler).

\section{Materials and Methods}

Isolation of purified, intact chloroplasts-Chloroplasts were obtained from $800 \mathrm{~g}$ of spinach leaves bought at the local market. The deribbed leaves were blended in a four-liter Waring blender for a total of 10 to $15 \mathrm{sec}$ in a chilled grinding medium ( $250 \mathrm{ml} / 100 \mathrm{~g}$ of leaves) containing $350 \mathrm{mM}$ sorbitol, $25 \mathrm{~mm}$ Mops, $2 \mathrm{mM}$ EDTA-Na $\mathrm{m}_{2}$ and $2 \mathrm{~mm}$ isoascorbate adjusted to $\mathrm{pH} 7.6$ with $\mathrm{KOH}$. After filtration on 6 layers of muslin and one layer of cheesecloth, the resulting filtrate was centrifuged at $2,100 \times g$ (Sorvall GSA rotor) for $1 \mathrm{~min}$. Each pellet was resuspended in $10 \mathrm{ml}$ of grinding medium and layered on top of a $40 \%$ Percoll solution containing the same ingredients as the grinding medium. After centrifugation $(2,100 \times g$ in a Sorvall HB-4 rotor for $3 \mathrm{~min}$ ), the pellets of purified chloroplasts were resuspended in the grinding medium and centrifuged at $2,000 \times g$ (Sorvall SS-34 rotor) for $3 \mathrm{~min}$ to wash out the residual Percoll.

Purification of envelope membranes-The fractionation of the chloroplasts into soluble and membrane proteins was achieved mainly according to Keegstra and Yousif (1986). The purified chloroplasts were resuspended in hypertonic medium and the chlorophyll adjusted at $2.5 \mathrm{mg}$ per ml (Bruinsma 1961). After a 15 min incubation on ice, the chloroplasts were ruptured with a Dounce homogenizer and diluted with 2 vol of TE buffer ( $10 \mathrm{mM}$ Tricine-NaOH, $\mathrm{pH} 7.6,2 \mathrm{mM}$ EDTA-Na ${ }_{2}$. The thylakoids were sedimented by a three $10 \mathrm{~min}$ steps differential centrifugation at $4,500,13,500$ and $23,500 \times g$ in a Sorvall $\mathrm{HB}-4$ rotor using slow acceleration. This centrifugation procedure led to a substantial increase in the envelope membrane yield. The yellow supernatant was layered on top of a discontinuous sucrose gradient consisting of $8 \mathrm{ml}$ of each of $1 \mathrm{M}, 0.65 \mathrm{M}$ and $0.4 \mathrm{M}$ sucrose solutions buffered with $\mathrm{TE}+\mathrm{MgCl}_{2}(5 \mathrm{mM})$ in the presence of $1 \mathrm{mM}$ PMSF and centrifuged for $6 \mathrm{~h}$ at $100,000 \times g$ in a Beckman SW-27 rotor. The stroma was recovered at the top of the gradient. The outer and inner envelope membrane fractions, found at the sucrose interfaces, were collected, diluted with TE + PMSF and sedimented at $100,000 \times \mathrm{g}$ for $60 \mathrm{~min}$ in a SW-27 rotor. After resuspension in TE + PMSF, the protein content was measured (Bradford 1976).

Separation of stroma, thylakoid and envelope membrane proteins into hydrophobic and hydrophilic fractions -The proteins of the stroma, the thylakoid and the inner and outer envelope membranes were separated according to their hydrophobicity and hydrophilicity using the properties of Triton X-114 as described by Bordier (1981). The original method was slightly modified: in order to avoid excessive loss of proteins, no sucrose cushion was used between the aqueous and the detergent phases; the detergent phase was extracted twice and its surface washed to eliminate contamination from the soluble proteins. The protein sample $(100 \mu \mathrm{g})$ was adjusted to $1 \% \mathrm{TX}-114$ with a solution containing $2 \%$ of precondensed TX-114, $10 \mathrm{~mm}$ Tris- $\mathrm{HCl}, \mathrm{pH} 7.4$ and $150 \mathrm{~mm} \mathrm{NaCl}$ and solubilized for $10 \mathrm{~min}$ at $0^{\circ} \mathrm{C}$. To allow condensation, the sample was then incubated for $10 \mathrm{~min}$ at $30^{\circ} \mathrm{C}$ in a water bath before being centrifuged for $3 \mathrm{~min}$ at $300 \times \mathrm{g}$ in a swinging bucket rotor (Universal Hettich 1200) at room temperature. From then on, the two phases were treated separately and the extraction was repeated once for the detergent phase and twice for the aqueous one. The surface of the detergent phase was washed with $500 \mu \mathrm{l}$ of buffer. The proteins of the two phases of interest were precipitated overnight in ice cold $80 \%$ acetone at $-20^{\circ} \mathrm{C}$. They were then centrifuged for $10 \mathrm{~min}$ at $10,500 \times \mathrm{g}$ in a Sorvall $\mathrm{HB}-4$ rotor before being prepared for isoelectric focusing or SDS-polyacrylamide gel electrophoresis (SDS-PAGE).

Electrophoresis and autoradiography-The precipitated proteins were denatured in boiling water for $3 \mathrm{~min}$ in 
$2 \%$ SDS, $0.005 \%$ bromophenol blue (w/v), $6.25 \%$ glycerol and $5 \%$ mercaptoethanol $(\mathrm{v} / \mathrm{v})$ in $0.0625 \mathrm{M}$ Tris- $\mathrm{HCl}$, $\mathrm{pH}$ 6.8. The samples were electrophoresed essentially according to Laemmli (1970) for 5 to $6 \mathrm{~h}$ on slab gels containing either $12 \%$ acrylamide throughout or a linear 10 to $18 \%(\mathrm{w} / \mathrm{v})$ acrylamide gradient accompanied by a 5 to $15 \%$ (w/v) sucrose gradient. The acrylamide to $N, N$ methylenebisacrylamide ratio was $30: 0.8$. The gels were fixed in a destaining solution containing $25 \%$ denatured ethanol and $8 \%$ acetic acid. They were either stained in $0.25 \%$ Coomassie brilliant blue R-250 (w/v) in 50\% methanol and $10 \%$ acetic acid $(\mathrm{v} / \mathrm{v})$ or as described by Neuhoff (1988). After destaining, they were dried under vacuum. Prior to drying, the bands of the 10 to $18 \%$ gradient acrylamide gel were scanned at $600 \mathrm{~nm}$ in a Zeiss-Disc ZK4 gel scanner and apparent mol wt estimated by calculating the relative mobility of the polypeptides compared to the following calibration proteins: phosphorylase $b$ ( $94 \mathrm{kDa})$, bovine serum albumin $(67 \mathrm{kDa})$, ovalbumin $(43 \mathrm{kDa})$, carbonic anhydrase $(30 \mathrm{kDa})$, soybean trypsin inhibitor $(20.1 \mathrm{kDa})$ and $a$-lactalbumin $(14.4 \mathrm{kDa})$. Autoradiographs were prepared on Kodak X-Omat AR films.

Isoelectric focusing-The organic and aqueous samples, obtained from an initial amount of $300 \mu \mathrm{g}$ of protein, were solubilized as described by Dunbar (1987). The isoelectric focusing procedure and $\mathrm{pH}$ determination were essentially according to Siegenthaler and Nguyen (1983). The gels were $7 \mathrm{~cm}$ long and contained $2 \%$ (v/v) TX-100 instead of Nonidet-P40. At the end of the run, the gels were treated as described by Jäckle (1979) before being submitted to the second dimension on a $12 \%$ SDS-gel.

Western blotting-After SDS-PAGE, the polypeptides were transferred to cellulose nitrate sheets using a semi-dry electroblotting apparatus (Sartorius). The electrophoretic buffer contained $48 \mathrm{~mm}$ Tris, $39 \mathrm{~mm}$ glycine, $1.3 \mathrm{~mm}$ SDS and $20 \%(\mathrm{v} / \mathrm{v})$ methanol ( $\mathrm{pH}$ at about 9$)$ (Heegaard and Bjerrum 1986).

Immunological studies-The control and TX-114 organic and aqueous phase fractions of the thylakoid, stroma and envelope polypeptides were challenged with several antibodies by the method of Towbin et al. (1979), except that 10 and $3 \%$ bovine serum albumin were replaced by 5 and $1 \%$ powdered milk and that no carrier serum was used. The secondary antibody directed against the first antiserum was labelled with alkaline phosphatase (KPL Laboratories) and used as described by Leary et al. (1983). Antibodies raised against the ribulose 1,5-bisphosphate carboxylase/oxygenase were kindly provided by Dr. A. Radunz.

\section{Results and Discussion}

Although the electrophoretic patterns of the four purified chloroplast fractions, stroma (S), thylakoids ( $T$ ), inner (IM) and outer (OM) envelope membranes, are differ- ent, there are some comigrating polypeptides which may be either polypeptides with identical Mr but different physicochemical properties or the result of some cross-contamination. Furthermore, the stroma can be expected to contain polypeptides of nucleic origin which accumulate there when their insertion into the thylakoid membranes is prevented (Cline et al. 1989). Polypeptides of chloroplastic origin can also be expected to accumulate there.

To investigate these possibilities, we separated the hydrophobic from the hydrophilic polypeptides in each of these fractions, using the Triton $\mathrm{X}-114$ phase partition method.

For sake of clarity, it is necessary to remind (Bennett 1982) that membrane proteins have been classified as integral or peripheral proteins. Integral membrane proteins can be defined as being "globular" or "fibrous". The "globular" proteins, such as a membrane-spanning protein, have a significant portion of their mass within the bilayer, while the "fibrous" ones have most of their mass protuding from the membrane and a hydrophobic polypeptide tail dipping into the membrane bilayer. Peripheral proteins, on the other hand, are membrane bound through non-covalent, probably mainly electrostatic interactions with the hydrophilic regions of integral proteins or with headgroup regions of the lipid bilayer. Once separated from the membrane, these proteins behave as soluble proteins and will therefore be recovered in the detergent-free phase.

From the definition given above, it is expected that "globular" proteins will partition in the detergent phase, while the "fibrous" ones, depending on the relative importance of their hydrophobic tail, may have an ambiguous behaviour and be recovered in the two phases.

\section{TX-114 phase partition: extraction conditions}

In his original procedure, Bordier (1981) recommended one extraction of the organic phase and three for the hydrophilic one. However, preliminary results showed that two extractions of the detergent phase were necessary to achieve a complete removal of the hydrophilic polypeptides.

\section{SDS-PAGE analysis of the four TX-114-treated fractions}

Introduction-Four fractions (T, S, IM and OM) were prepared and then submitted to TX-114 extraction. The electrophoretic patterns of the resulting aqueous and organic phase polypeptides, along with their corresponding controls, are shown in Fig. 1. Although each phase contained its own set of polypeptides, there were still several similarities in the electrophoretic mobilities. Among the polypeptides having the same electrophoretic mobility, some had a similar behaviour towards TX-114 treatment and might therefore be identical, while others did not partition in the same phase, thus indicating their different 
nature. As no chlorophyll could be detected in the stroma or the envelope membranes, contamination by thylakoid components could be ruled out. However, stroma material, arising from inner membrane-derived vesicles, can be present in the envelope fractions (Cremers et al. 1988) as well as in the thylakoid one.

Membrane phase partition-The complexity of the electrophoretic patterns of the membrane polypeptides, especially those of the envelope membranes (IMc, OMc), can be partly overcome by separating these polypeptides according to their hydrophobic and hydrophilic properties. For instance, one can see (Fig. 1, IMo, OMa) that most of the inner membrane polypeptides were recovered in the organic phase while those of the outer membrane partitioned preferentially in the aqueous phase. On close examination, about half of the envelope membrane polypeptides were recovered in only one of the two phases. The partitioning of the polypeptides was therefore quite good whenever strongly hydrophobic or strongly hydrophilic polypeptides were concerned. Indeed, the inner membrane 94,34 and $33 \mathrm{kDa}$, the outer membrane 76, 66 and $22 \mathrm{kDa}$ polypeptides, the thylakoid LHCP and Cyt. f (as localized also by Bricker and Sherman 1982) as well as several thylakoid low Mr polypeptides were exclusively recovered in the organic phases (Fig. 1, IMo, OMo and To). In contrast, the thylakoid $30 \mathrm{kDa}$ and the outer membrane 109 , 40, 24, 23 and $21 \mathrm{kDa}$ polypeptides (Fig. 1, Ta and OMa) were only encountered in the aqueous phase. Consequently, by discriminating between hydrophobic and hydrophilic polypeptides, TX-114 enhanced the SDS-PAGE resolution.

Other polypeptides were common to the two phases and their characterization as integral ("globular" and "fibrous") or peripheral is more difficult. As there are several answers to that situation, complementary methods have to be used. Here, we may be dealing with polypeptides that were not totally removed from the membrane (Bordier 1981). These polypeptides could be "fibrous" proteins, the solubilization of which depends strongly on the nature of their hydrophobic tail and its interactions with the membrane. Such may be the case of the IM81 and $70 \mathrm{kDa}$ polypeptides which were found simultaneously in both the organic and aqueous phases, as revealed by $2 \mathrm{D}$ electrophoresis (see arrows in Fig. 2A and B). However, the $a$ and $\beta$ subunits of $C_{1}$, known as being hydrophilic polypeptides, failed to partition completely in the aqueous phase (see Figs. 1 and 3, To, a). As suggested by Bricker and Sherman (1982), a small amount of $C_{1}$ may remain attached to $\mathrm{CF}_{0}$ or, as these polypeptides are very abundant, there could be a spilling over phenomenon. In other instances, we were dealing with two or more totally different polypeptides. Indeed, the thylakoid $15 \mathrm{kDa}$ band (Fig. 1 and Fig. 3A, Tc, o, a) partitioned unequally between the two phases. Figure 3B shows that it was in fact resolved into a hydrophobic polypeptide synthesized in the chloroplast (as attested by its ${ }^{35} \mathrm{~S}$-labelling in the organic phase) and a hydrophilic one of cytoplasmic origin (no labelling in the aqueous phase).

Comigration may also occur in one of the phases. As shown in Fig. 2C, a unique Coomassie band (Control: aqueous phase $72 \mathrm{kDa}$ ) may contain two polypeptides having different pI (see arrows). In this case, they were not charge isomers but again polypeptides of different genomic origin as shown by proteosynthesis experiments (results not shown).

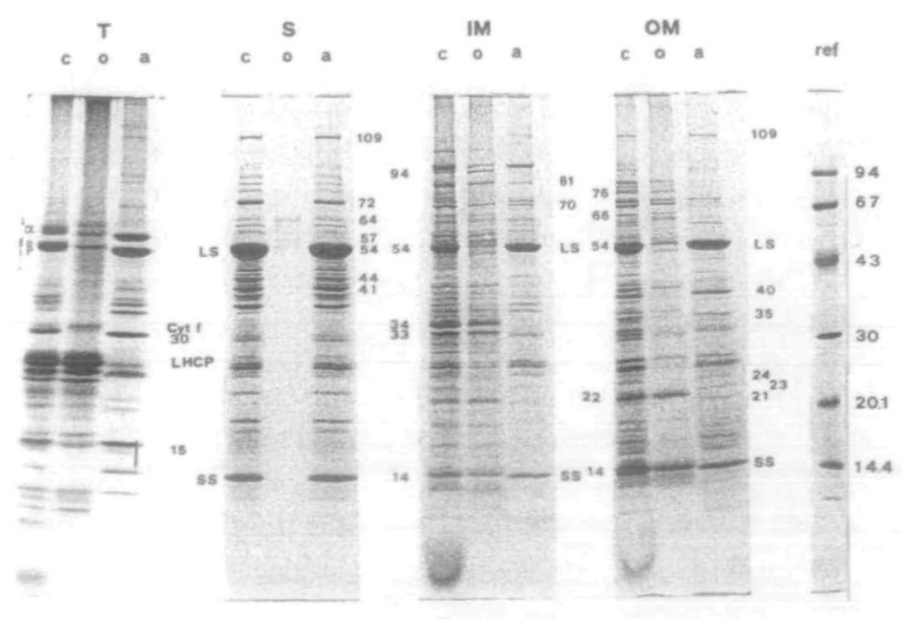

Fig. 1 Electrophoretic separation of thylakoid, stroma, inner and outer envelope membrane polypeptides. The TX-114 phases were extracted twice and the aqueous phases three times. The references (ref) and the separating gel containing a linear 10-18\% acrylamide gradient are as described in Materials and Methods. Each control fraction (c) contained $30 \mu \mathrm{g}$ of untreated polypeptides. The organic (o) and aqueous (a) fractions were obtained from an initial amount of $100 \mu \mathrm{g}$ of proteins. T, thylakoid; $S$, stroma; IM, inner membrane; OM, outer membrane. Apparent mol wt values are indicated in $\mathrm{kDa}$. 

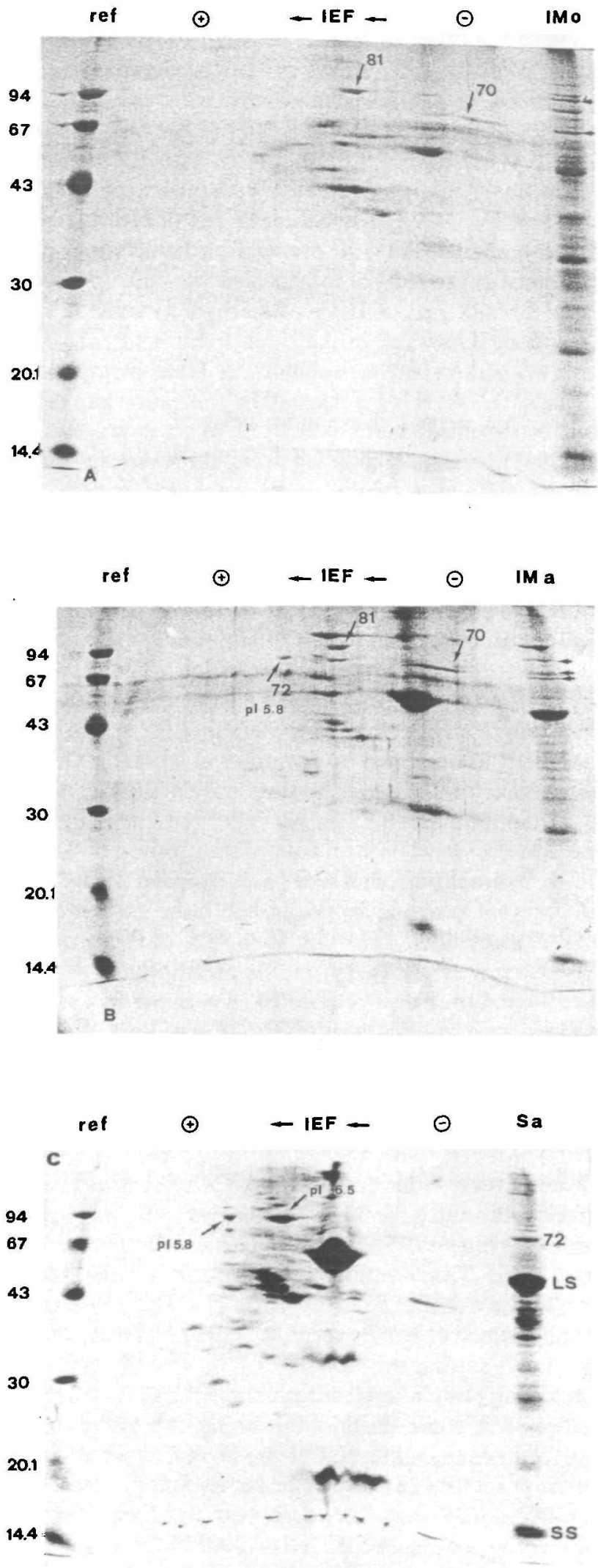

Stroma phase partition-All but five of the stroma polypeptides were recovered in the aqueous phase (Fig. 1 $\mathrm{Sa}$. The presence of Coomassie bands in the organic phase (Fig. 1 So) was rather unexpected, but nevertheless, TX-114 phase partitioning was highly reproducible. These faint bands corresponded to polypeptides having $\mathrm{Mr}$ of 64 , $57,54,44$ and $41 \mathrm{kDa}$ as determined by densitometric tracings (results not shown). Since bands of equivalent $\mathrm{Mr}$ could be seen in the organic phases of the membrane fractions, envelope and/or thylakoid components might have contaminated the stroma. But, if contamination by membrane material occurred, one would have expected to find all or at least most of the membrane hydrophobic polypeptides in the stroma organic phase (Fig. 1 So). Since it was obviously not the case, one can reasonably exclude any significant contamination of the stroma fraction by the envelope or thylakoid membranes. Furthermore, the stroma was unlikely to be contaminated by thylakoids since the stromal fraction did not contain any trace of chlorophyll. The $63 \mathrm{kDa}$ band was doubtful as it comigrated with an artifact sometimes present in our electrophoreses. The presence of these hydrophobic proteins is best explained by assuming that we are dealing with transit polypeptides of chloroplastic (S57, $54 \mathrm{kDa}$ ) or nuclear (S44, $41 \mathrm{kDa}$ ) origin, as suggested by proteosynthesis experiments (results not shown) and by Cline et al. (1989). Indeed, it is expected that these polypeptides, the fate of which is to be eventually integrated in a membrane, contain several hydrophobic domains which would favour an organic phase-partitioning.

Peripheral membrane proteins or soluble contaminants?-Another interesting feature of the polypeptide separation in the presence of TX-114 was the unexpectedly great number of envelope membrane polypeptides having a hydrophilic character (Fig. $1 \mathrm{IMa}, \mathrm{OMa}$ ). These may be peripheral (loosely membrane-bound) polypeptides or stroma components. The latter was indeed the case with the large and small subunits of the RubisCO which are known to copurify with the envelope membranes (Pineau et al. 1979, Joyard et al. 1982). Such was also the case with the inner membrane $72 \mathrm{kDa}$ (Fig. 2B: pI 5.8) which, when present, reacted with the antibody against the stroma $72 \mathrm{kDa}$ raised in our laboratory (data not shown). On the other hand, $O M 35,23$ and 21, found exclusively in the membrane aqueous phase, did not have any counterpart in the stroma (Fig. $1 \mathrm{Sa}$ ). This strongly suggests that these outer membrane polypeptides are true peripheral com-

Fig. 2 2-Dimension electrophoretic separation of the organic (A) and the aqueous (B) phases of the inner membrane and the aqueous phase of the stroma (C). The IEF was as described in Materials and Methods and the separating gel of the second dimension contained $12 \%$ acrylamide. 


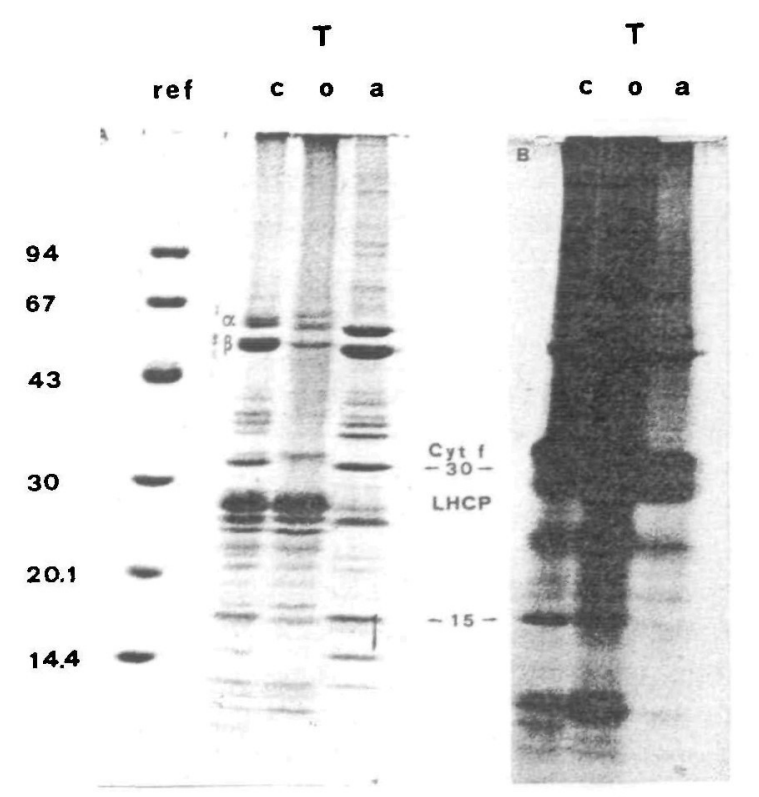

Fig. 3 Electrophoretic separation of labelled thylakoid polypeptides after TX-114 treatment (A) and its autoradiography (B). Experimental conditions and symbols as in Fig. 1. For proteosynthesis in organello, intact chloroplasts were resuspended ( $1 \mathrm{mg} \mathrm{chl} / \mathrm{ml}$ ) in an incubation medium containing $100 \mathrm{mM} \mathrm{KCl}$, $2 \mathrm{mM} \mathrm{MgCl}$ and $66 \mathrm{mM}$ Tricine- $\mathrm{KOH}$ at $\mathrm{pH}$ 7.6. The light-driven incorporation of ${ }^{35} \mathrm{~S}$-methionine $(150 \mu \mathrm{Ci} / \mathrm{mg}$ chl; specific activity $>1,000 \mathrm{Ci} / \mathrm{mmol}$; SJ 1515 , Amersham) was performed in a water bath at $20^{\circ} \mathrm{C}$ for $60 \mathrm{~min}$.

ponents of the envelope. It is thus clear that the preliminary separation obtained through the use of the TX114 phase partition succeeds in eliminating all the stroma trapped in the inner or outer envelope membrane vesicles. The organic phase polypeptides are thus exclusively of membrane origin, while those found in the aqueous phase may still have a dual origin, namely membrane and stroma.

Proteins revealed by $T X-114$ phase partition-It is noteworthy that the use of this technique allowed the visualization of polypeptides which have close $\mathrm{Mr}$ and are normally partly overlapping. This was most obvious near the large subunit (LS) of the RubisCO. Indeed, a $54 \mathrm{kDa}$ polypeptide was found in the organic phases of the stroma, of the inner and outer envelope membranes at the level of the RubisCO (Fig. 1 So, IMo, OMo). The RubisCO, in its $18 \mathrm{~S}$ holoenzyme form, is known to remain bound to the envelope membranes during their purification (Joyard et al. 1982) and has been, so far, impossible to eliminate (Pineau et al. 1979, Werner-Washburn et al. 1983). To assess the nature of this $54 \mathrm{kDa}$ organic band, an antibody raised against the RubisCO was tested on the stroma, inner and outer envelope membrane TX-114-treated fractions (S, IM and $\mathrm{OM}$ ) and their respective controls. The results are shown in Fig. 4. A positive reaction was found only in the controls and the aqueous phases of the three fractions. No reaction could be detected on any of the organic phases. Furthermore, a polyclonal antibody was raised in our laboratory against the stromal organic phase $54 \mathrm{kDa}$ polypeptide. When tested, it did not react with LS in any of the aqueous phases but reacted with all three organic phases (see Fig. 5). The rather weak reaction of the controls can be explained by the presence of the LS which represents most of the $30 \mu \mathrm{g}$ of total protein content. There is, therefore, a fair probability that the hydrophobic $54 \mathrm{kDa}$ polypeptide, unmasked in the three fractions by TX-114 treatment, is not the large subunit. It is noteworthy that the RubisCO, which is not released by sonication and other drastic treatments (Joyard et al. 1982, Werner-Washburne et al. 1983), can be eliminated from the envelope membranes by TX-114 phase partition. Moreover, the RubisCO small subunit band in the IM and OM fractions was split between the organic and aqueous phases. Fig. 4 shows that the polypeptide with hydrophobic behaviour was not the small subunit (SS) as there was no immunological reaction with the RubisCO antibody.

Solubilization versus phase partition-The complex mixture of membrane proteins can also be fractionated by solubilizing them in chloroform/methanol $(2: 1 \mathrm{v} / \mathrm{v})$ or in $0.1 \mathrm{~N} \mathrm{NaOH}$ as described by Joyard et al. (1982). On the basis of their results, these authors concluded that 55 to $60 \%$ of the total envelope polypeptides are integral membrane proteins, most of them being characterized by a high Mr. A hydrophobic character was assigned to half of these integral proteins by virtue of their chloroform/ methanol solubility. The other 40 to $45 \%$ of the envelope polypeptides were released from the membrane by $\mathrm{NaOH}$ treatment and were thus considered as peripheral proteins. The above two solubilization methods may lead, through delipidation or limited saponification of lipids, to a total or partial destruction of the membrane. Furthermore, the quaternary and tertiary conformations of the proteins are probably not preserved, leading to the exposure of hydrophilic and hydrophobic domains which may be erroneously determinant towards the final solubilization and the characterization as intrinsic or extrinsic. This drawback does not happen with TX-114 phase partition, which has been shown by the works of Sanchez-Ferrer (1989a, b, 1990) and Soll and Bennett (1988) to keep the proteins in their native state, thus allowing the isolation of functional enzymes. The resulting protein separations obtained by TX-114 solubilization and phase partitioning or by solubilization in chloroform/methanol or $\mathrm{NaOH}$ are in fact based on very different properties and cannot be compared. Indeed, the polypeptides E37 and E24 described by Joyard et al. (1982), corresponding respectively to our IM34 and OM22 (Fig. 1, IMo, OMo) were not solubilized in chloroform/methanol but partitioned in TX-114 as integral poly- 


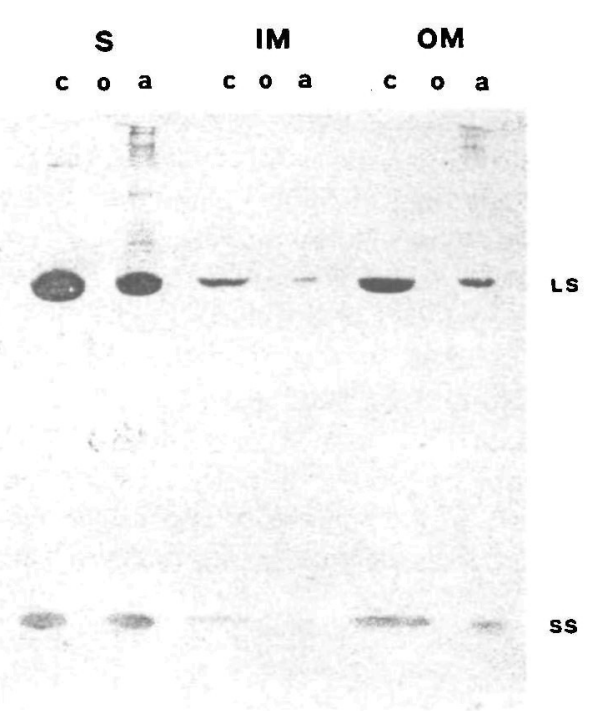

Fig. 4 Western blot of stroma, inner and outer envelope membrane polypeptides after treatment with TX-114 and separation on a $12 \%$ acrylamide gel slab. Antibodies against the RubisCO (LS, SS) were tested. Symbols are as in Fig. 1.

peptides. However, the two results taken together could very well indicate that E37 and E24 are "fibrous" proteins according to the definition given above. However, it is interesting that the E14 (corresponding to our IM14 and OM14) which was not totally extracted by chloroform/methanol (Joyard et al. 1982), was also found split between the aqueous and organic fractions of the inner and outer membranes (Fig. $1 \mathrm{IMa}, \mathrm{o}$ and $\mathrm{OMa}, \mathrm{o}$ ). As we have shown, theses organic bands were not related to the RubisCO small subunit as they did not react with the RubisCO antibody (Fig. 4). It is also likely that the faint band (at the E54 level), found by Joyard et al. (1982) in the chloroform/methanol-soluble fractions from stroma and envelope, is identical to the one found in our stroma and membrane organic fractions, and which was shown to be different from the RubisCO LS (Figs. 1, 4 and 5).

Conclusions-To decrease the complexity of the electrophoretic patterns of the envelope membrane polypeptides, it is thus advisable to fractionate the total set of proteins prior to electrophoresis. The TX-114 phase partition method, which is highly reproducible and easy to handle, has been shown here to be a valuable tool for chloroplast envelope membranes. It provides a physico-chemical environment suitable for membrane protein purification and allows the separation of integral proteins from peripheral and soluble ones.

Furthermore, the organic phases of the inner and outer envelope membranes contain "globular" proteins probably involved in chloroplast transport and communication. Moreover, the two RubisCO subunits, which are

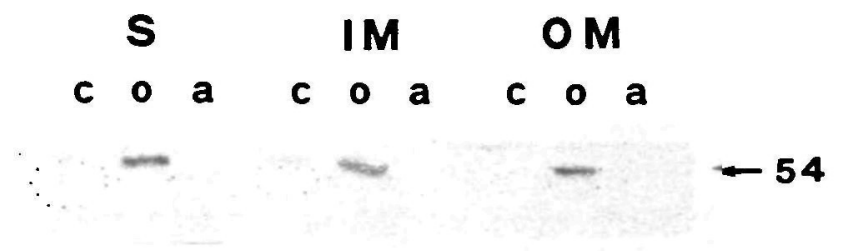

Fig. 5 Antibodies against the hydrophobic $54 \mathrm{kDa}$ were tested on a Western blot as described in Fig. 4. Only the relevant part is shown. The antigen was purified on SDS gel electrophoresis. The desired gel bands were pooled, crushed in liquid nitrogen and dissolved in the complete Freund's adjuvant diluted $1: 1$ with distilled water. Three injections were made intradermically at 2 to 3 weeks intervals in a white New Zealand rabbit. Symbols are as in Fig. 1.

always present in the three membraneous fractions, cannot be dislodged from the two envelope membranes unless TX114 phase partition is used. However they are easily washed out of the thylakoid. This suggests (as also proposed by Joyard et al. 1982) that the RubisCO could be specifically associated with the chloroplast envelope.

Therefore, the ability of TX-114 to separate membrane proteins according to their relative hydrophobicity while preserving their native form is a potentially very powerful method towards the characterization and purification of envelope chloroplast polypeptides.

The authors thank Miss Cécile Seuret and M. Daniel Leemann for their able technical assistance and Dr. A. Radunz for his generous gift of RubisCO antibody. This work is part of a doctoral program which is being carried out by N.D. in the Laboratoire de Physiologie végétale, Université de Neuchâtel.

\section{References}

Bennett, J. P. (1982) Solubilisation of membrane-bound enzymes and analysis of membrane protein concentration. In Techniques in Lipid and Membrane Biochemistry, Part 1, B408. pp. 1-22. Elsevier/North-Holland Scientific Publishers Ltd, County Clare, Ireland.

Bordier, C. (1981) Phase separation of integral membrane proteins in Triton X-114 solution. J. Biol. Chem. 256: 1604-1607.

Bradford, M. (1976) A rapid and sensitive method for the quantitation of microgram quantities of protein utilizing the principle of protein-dye binding. Anal. Biochem. 72: 248-254.

Bricker, T. M. and Sherman, L. A. (1982) Triton X-114 phasefractionation of maize thylakoid membranes in the investigation of thylakoid protein topology. FEBS Lett. 149: 197-202.

Bruinsma, J. (1961) A comment on the spectrophotometric determination of chlorophyll. Biochim. Biophys. Acta 52: 576578. 
Cline, K., Keegstra, K. and Staehelin, L. A. (1985) Freeze-fracture electron microscopic analysis of ultrarapidly frozen envelope membranes on intact chloroplasts and after purification. Protoplasma 125: 111-123.

Cline, K., Fulsom, D. R. and Viitanen, P. V. (1989) An imported thylakoid protein accumulates in the stroma when insertion into thylakoids is inhibited. J. Biol. Chem. 264: 14225-14232.

Cremers, F.F.M., Voorhout, W.F., van der Krift, T.P., Leunissen-Bijvelt, J. J. M. and Verkleij, A. J. (1988) Visualization of contact sites between outer and inner envelope membranes in isolated chloroplasts. Biochim. Biophys. Acta 933: 334-340.

Douce, R., Block, M. A., Dorne, A.-J. and Joyard, J. (1984) The plastid envelope membranes: their structure, composition, and role in chloroplast biogenesis. Subcell. Bioch. 10: 1-86.

Dumont, N., Bovet, L. and Siegenthaler, P. A. (1988) Separation of stromal and envelope membrane proteins of spinach chloroplasts into hydrophilic and lipophilic fractions. Experientia 44: 44.

Dumont, N. and Siegenthaler, P. A. (1989) Separation of stromal, thylakoid, inner and outer envelope membrane proteins of spinach chloroplasts into hydrophilic and hydrophobic fractions. In Current Research in Photosynthesis. Proceedings of the VIIIth International Conference on Photosynthesis. vol 3: 14.849. Edited by Baltscheffsky. Kluwer Academic Publishers.

Dunbar, B. S. (1987) Sample preparation and solubilization for one-dimensional polyacrylamide gel electrophoresis and isoelectric focusing. In Two-Dimensional Electrophoresis and Immunological Techniques. p. 217. Plenum Press, New York.

Heegaard, N. H. H. and Bjerrum, O. J. (1986) In Handbook of Immunoblotting of Proteins. Edited by Bjerrum, O. J. and Heegaard, H. H. CRC Press, Boca Raton, Florida.

Jäckle, H. (1979) Visualization of proteins after isoelectric focusing during two-dimensional gel electrophoresis. Anal. Biochem. 98: 81-84.

Joyard, J., Grossman, A., Bartlett, S. G., Douce, R. and Chua, N.-H. (1982) Characterization of envelope membrane polypeptides from spinach chloroplasts. J. Biol. Chem. 257: 10951101.

Keegstra, K. and Yousif, A. E. (1986) Isolation and characterization of chloroplast envelope membranes. Methods Enzymol. 118: 316-325.
Kjellbom, P., Larsson, C., Rochester, C. P. and Andersson, B. (1989) Integral and peripheral proteins of the spinach leaf plasma membrane. Plant Physiol. Biochem. 27: 169-174.

Laemmli, U. K. (1970) Cleavage of structural proteins during the assembly of the head of bacteriophage T4. Nature 227 , no 5259: 680-685.

Leary, J. J., Brigati, D. J. and Ward, D. C. (1983) Rapid and sensitive colorimetric method for visualizing biotin-labeled DNA probes hybridized to DNA or RNA immobilized on nitrocellulose: Bio-blots. Proc. Natl. Acad. Sci. USA 80: 4045-4049.

Neuhoff, V., Arold, N., Taube, D. and Ehrhardt, W. (1988) Improved staining of proteins in polyacrylamide gels including isoelectric focusing gels with clear background at nanogram sensitivity using Coomassie brilliant blue G-250 and R-250. Electrophoresis 9: 255-262.

Pineau, B., Ledoigt, G., Maillefer, C. and Lefort-Tran, M. (1979) Présence de sous-unités de la RubPcase dans les enveloppes des chloroplastes d'épinard. Plant Sci. Lett. 25: 331-343.

Sanchez-Ferrer, A., Villalba, J. and Garcia-Carmona, F. (1989a) Triton X-114 as a tool for purifying spinach polyphenol oxidase. Phytochemistry 28: 1321-1325.

Sanchez-Ferrer, A., Bru, R. and Garcia-Carmona, F. (1989b) Novel procedure for extraction of a latent grape polyphenoloxidase using temperature-induced phase separation in Triton X-114. Plant Physiol. 91: 1481-1487.

Sanchez-Ferrer, A., Bru, R. and Garcia-Carmona, F. (1990) Partial purification of a thylakoid-bound enzyme using temperature-induced phase partitioning. Anal. Biochem. 184: 279282.

Siegenthaler, P. A. and Nguyen, T. D. (1983) Proteins and polypeptides of envelope membranes from spinach chloroplasts. Biochim. Biophys. Acta 722: 226-233.

Soll, J. and Bennett, J. (1988) Localization of a 64-kDa phosphoprotein in the lumen between the outer and inner envelopes of pea chloroplasts. Eur. J. Biochem. 175: 301-307.

Towbin, H., Staehelin, T. and Gordon, J. (1979) Electrophoretic transfer of proteins from polyacrylamide gels to nitrocellulose sheets: Procedure and some applications. Proc. Natl. Acad. Sci. USA 76: 4350-4354.

Werner-Washburn, M., Cline, K. and Keegstra, K. (1983) Analysis of pea chloroplast inner and outer envelope membrane proteins by two-dimensional gel electrophoresis and their comparison with stromal proteins. Plant Physiol. 73: 569-575.

(Received April 2, 1990; Accepted August 28, 1990) 